\title{
An Anthropomorphic Polyvinyl Alcohol Triple-Modality Brain Phantom Based on Colin27
}

\author{
Sean Jy-Shyang Chen ${ }^{1}$, Pierre Hellier ${ }^{2}$, Jean-Yves Gauvrit ${ }^{2,3,4}$, \\ Maud Marchal ${ }^{2}$, Xavier Morandi ${ }^{2,3,4}$, and D. Louis Collins ${ }^{1}$ \\ 1 McConnell Brain Imaging Centre, Montreal Neurological Institute, \\ McGill University, Montreal, Canada \\ sjschen@bic.mni.mcgill.ca \\ 2 INRIA, Centre de Recherche, Rennes, Bretagne Atlantique, France \\ 3 INSERM U746, IRISA, F-35042, Rennes, France \\ 4 Université de Rennes 1, 35065, Rennes, France
}

\begin{abstract}
We propose a method for the creation of an anatomically and mechanically realistic brain phantom from polyvinyl alcohol cryogel (PVAC) for validation of image processing methods for segmentation, reconstruction, registration, and denoising. PVA-C is material widely used in medical imaging phantoms for its mechanical similarities to soft tissues. The phantom was cast in a mold designed using the left hemiphere of the Colin27 brain dataset [1] and contains deep sulci, a complete insular region, and an anatomically accurate left ventricle. Marker spheres and inflatable catheters were also implanted to enable good registration and simulate tissue deformation, respectively. The phantom was designed for triple modality imaging, giving good contrast images in computed tomography, ultrasound, and magnetic resonance imaging. Multimodal data acquired from this phantom are made freely available to the image processing community (http://pvabrain.inria.fr) and will aid in the validation and further development of medical image processing techniques.
\end{abstract}

\section{Introduction}

The human cerebrum is a topologically complex organ with deep fissures and sulci over its lateral and medial surfaces, as well as fluid filled ventricles of complex form in its interior. The creation of a physical model capable of depicting the form of the cerebrum in a realistic manner is not trivial due in part to these deep structures. Previous works in creating brain phantoms have either reduced the depth of the sulci [2], or only recreated the brain's form superficially with dessert gelatin molds 34]. Although these phantoms bear a gross cursory resemblance to the human cerebrum, they do not accurately depict its anatomy. Registering these phantoms to their acquired multi-modality images may also not be straight-forward since the landmarks on the phantom are not easy to find or image. This may be due to the structures being smaller than the image resolution or having insufficient contrast of the markers with respect to the surrounding

T. Jiang et al. (Eds.): MICCAI 2010, Part II, LNCS 6362, pp. $92-100,2010$.

(C) Springer-Verlag Berlin Heidelberg 2010 


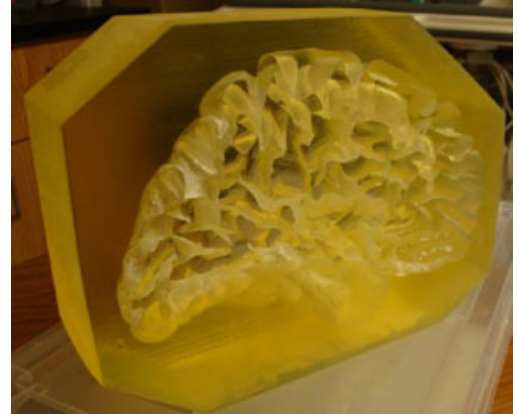

(a)

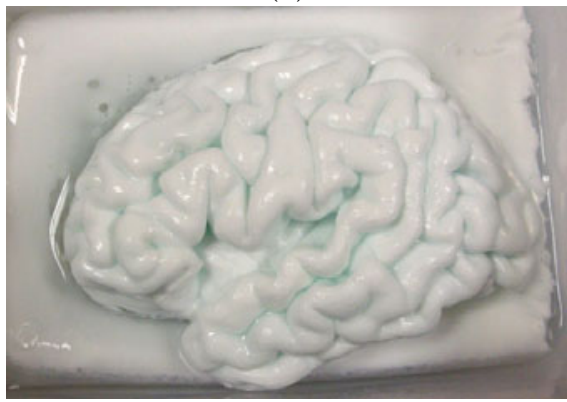

(c)

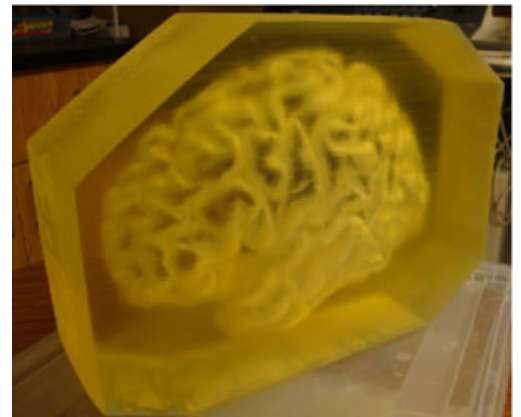

(b)

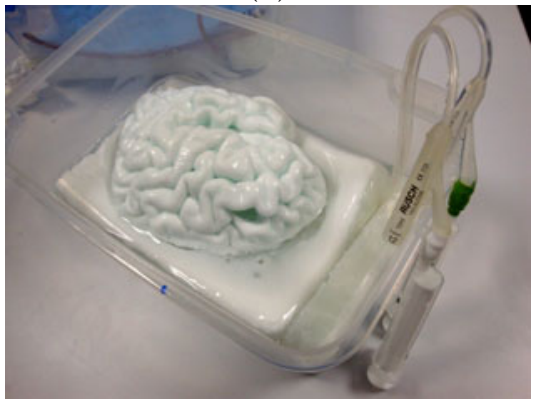

(d)

Fig. 1. Two views of the elastic Colin 27 based brain phantom mold from (a) the inside and (b) the outside. (c) The PVA-C phantom casted from our mold using our PVA solution recipe. Note the deep sulci and insular regions of the phantom. (d) The setup for scanning the phantom with the catheters used to inflate the phantom on the right.

tissues. For instance, Reinertsen and Collins [4] rely on the presence of bubbles in their phantom to act as landmarks for validation.

Our goal is to create a triple modality human brain phantom containing anatomically realistic structures and physically realistic texture. The phantom was then scanned and the multimodal images are made publically available. Polyvinyl alcohol was selected for phantom construction.

Polyvinyl alcohol (PVA) is a synthetic polymer synthesized from polyvinyl acetate through hydrolysis of the latter's acetate groups [2]. When liquid PVA solutions undergo a specified period of freezing at a set temperature and is then allowed to slowly thaw to room temperature, this freeze-thaw cycle (FTC) transforms the liquid PVA solution into a elastic semi-opaque gel know as polyvinyl alcohol cryogel (PVA-C) 5678.

PVA-C is used in biomedical research for producing soft tissue phantoms in studies to develop, characterize, and refine different imaging or image processing methods 91011]. It is a good material for such studies since it has similar texture, mechanical properties such as compressibility and elasticity, and similar water content to many soft tissues 2491011/2 13]. PVA-C has been used in 
the construction of phantoms for studying a wide variety of tissues including that of the heart [14, breast [1011], prostate [13], arterial vasculature 82, the brain [42], as well as their abnormal tissues in the form of lesions and tumours 101113 .

In the following sections, we describe the methods we used to build our brain phantom. The contributions of our phantom to the literature include:

Anatomical accuracy: Deep cortical structures of the Colin27 cerebrum, such as the sulci, the insular region and the ventricles are realistically represented in the cast phantom.

Realistic texture: Recipes of PVA-C with textures most similar to human cerebral tissues were determined through the feedback of an experienced neurosurgeon who knows the tactility of the human brain and tumour tissues.

Multimodal imaging: PVA-C formulations that can be imaged effectively with high contrast CT, US, and MR are used to construct the phantom and its implants.

Freely available data: Images acquired using the US, MR, and CT scanners are made available through our website to researchers and the general public. Images for this phantom were acquired using magnetic resonance imaging ( $\mathrm{T} 1$ and $\mathrm{T} 2$ weighted, PD, FLAIR, and DTI), ultrasound imaging, and computed tomography, to ensure that the phantom exhibits similar contrast to images of the live cerebrum acquired using these imaging modalities.

\section{Phantom Construction}

\subsection{PVA Solution Preparation}

The PVA solutions used to cast the brain phantom and its various components were prepared using 99-100\% hydrolyzed PVA with an average molecular weight of 86 kilodaltons (Code:418120010) from Acros organics (Geel, Belgium).

Master solutions of $5 \%$ and $8 \%$ mass percentage $(\mathrm{w} / \% \mathrm{w})$ PVA solutions were prepared by heating distilled water and adding a percentage weight of PVA to the water. The mixture was constantly stirred until the PVA particles were well hydrated upon which the holding vessel of PVA and water mixture was placed in an oven at $93^{\circ}-95^{\circ}$ Celsius for 7 hours. Small quantities of distilled water were added back into the solutions according to the amounts lost during preparation of the PVA solution. PVA solutions with different lower mass percentages can be subsequently produced by heating the master solutions and mixing in additional water.

\subsection{PVA-C Preparation}

PVA-C was prepared by completely freezing and thawing PVA solutions, which resulted in semi-translucent flexible gel. The solutions were placed in a room temperature $\left(25^{\circ}\right.$ Celsius $)$ chest freezer and cooled to $-25^{\circ}$ to $-20^{\circ}$ Celsius. After 12 hours of freezing at the aforementioned temperatures, the freezer was shut-off and its interior was allowed to rise back to room temperature over another 12 hour period. These freeze thaw cycles (FTC) were repeated as needed to vary the consistency of the produced PVA-C. 


\subsection{Brain Mold}

The mold for our brain phantom was based on the polygonal surface mesh segmented from the left hemisphere of the Colin27 data set [1]. This mesh was then subtracted from a rectangular prism mesh to create a "negative" of the cortical surface, which was then used for stereolithographic printing.

We utilized the services of RedEye On Demand (Eden Prairie, MN, USA) for the fabrication of our brain phantom mold using the TangoPlus Polyjet Resin (FC-930) as the material. This clear rubber-like photopolymer is disposited layer by layer in order to produce a finished three dimensional object (See Fig. 17). We found that the mold made using this material was able to accurately model the sulci and insular region of the cerebral hemisphere. It also has enough flexibility to allow demolding without damaging the PVA-C phantom and reverts itself to its original shape when deformed.

The bottom of a plastic tub was cut out and glued around the opening of the flexible rubber mold. This allows us to cast a base for our demolded phantom and limit its relative movement when placed and imaged in another plastic tub of the same size.

The mold component for the left ventricle of the phantom was constructed separately using silicone bathroom caulk. Layers of caulk approximately $2 \mathrm{~mm}$ thick were applied to vellum traces from life-size printouts of segmented $2 \mathrm{~mm}$ sagittal sections of the left ventricle. These layers were then assembled medially to laterally and aligned using cross-hairs on the printout traces to maintain placement accuracy of the sagittal sections and then covered with additional silicone rubber caulk to smooth the mold component surface.

\subsection{Approximating Live Brain Texture}

An array of PVA-C samples was prepared with either 1, 2, or 3 FTCs and $4 \%, 5 \%, 6 \%$, or $8 \%$ PVA solutions, producing 12 different PVA-C samples. The samples were palpated at room-temperature by a gloved neurosurgeon who is familiar with the texture of human brain and brain pathologies.

The experienced neurosurgeon was then asked to rate each sample from 0 to 10 with the former being nothing like live brain and the latter being exactly like live brain, while noting whether the sample was softer or firmer than healthy brain tissue. The neurosurgeon was then asked to choose 2 of the samples which felt most like low grade gliomas.

The surgeon rated the PVA-C sample created from $6 \%$ PVA solution at 1 FTC as being similar to palpating the surface of a live brain and the $4 \%$ PVA at 3 FTC as being similar to palpating a low grade glioma.

We measured the elastic modulus of the $6 \%$ PVA 1 FTC PVA-C sample using a 3369 Dual Column Testing System (Instron, Norwood, MA, USA) and found that it has a value $4.6 \mathrm{kPa}$, which is within the range found for human brain tissue [15]. 


\subsection{Implants}

To make our phantom useful for tests in image guidance and registration, structures created from various formulations of PVA-C were included into the phantom. Spherical registration marker were created using a harder PVA-C made from $8 \%$ PVA solution that has undergone 2 freeze-thaw cycles (FTC). These were molded using the containers for reflective passive spheres used in optical tracking and are approximately $12 \mathrm{~mm}$ in diameter. A PVA-C "tumour" was also created using $4 \%$ PVA solution with 2 FTC and then implanted into the phantom. The tumour was molded using the plastic case from inside a Kinder surprise (Ferrero, Pino Torinese, Italy). When the phantom is completed, the markers and the tumour will each have undergone 3 FTC.

These PVA-C structures are skewered and suspended using $0.45 \mathrm{~mm}$ monofilament fishing lines inside the phantom at the desired location. The left ventricle mold component was clamped and also suspended with fishing lines in a similar fashion.

Finally, we placed the inflatable head of a urinary catheter into the frontal lobe of the phantom and another in the medial portion of the phantom in the cast base of the mold. Each urinary catheter can be inflated with up to $10 \mathrm{ml}$ of water using a syringe in the manner described by Reinertsen and Collins [4] to vary the extent of deformation on the phantom.

\section{Triple Modality Imaging Contrast}

Commonly available chemicals were used to change the contrast of our phantom for imaging in US, MR, and CT. A PVA-C with the PVA concentration and FTC resembling textures similar to a living human cerebrum was chosen to be the base solution for dissolving the contrast enhancing chemicals.

To increase back-scattering of sound waves in US imaging, solutions containing talcum powder at $4 \%, 2 \%, 1 \%$, and $0.5 \%$ weight of the base solution were mixed. Each of the samples was immersed in water and imaged with a Sonosite 180 Plus (Sonosite, Bothell, WA, USA) diagnostic ultrasound system tracked using a Stealth neurosurgical station (Medtronic, Minneapolis, MN, USA) and visually examined for contrast with the surrounding water and implanted PVA markers spheres.

For increasing phantom contrast in CT imaging, a powdered barium sulphate $\left(\mathrm{BaSO}_{4}\right)$ preparation used for colon enema (Guerbet Micropaque Colon, Guerbet, Villepinte, Île-de-France, France) was mixed into our PVA solutions. Solutions were prepared with $8 \%, 6 \%, 3 \%$, and $1 \%$ weight $\mathrm{BaSO}_{4}$ of the initial base solution. PVA-C samples were made from each of these solutions and imaged using a LightSpeed 16 VCT scanner (GE Healthcare, Little Chalfont, Buckinghamshire, UK).

To enhance the signal in $\mathrm{T} 1$ weighted images, copper sulphate $\left(\mathrm{CuSO}_{4}\right)$ was added to the PVA mixture in small quantities. Minute quantities of $\mathrm{CuSO}_{4}$ dramatically increase the contrast of the PVA sample in T1 and T2 weighted images. To find an optimal concentration of $\mathrm{CuSO}_{4}$, we prepared $0.2 \%, 0.1 \%$, 
0.05\%, and $0.025 \%$ anhydrous $\mathrm{CuSO}_{4}$ PVA-C samples and imaged them with $\mathrm{T} 1$ and T2 imaging sequences on a Verio 3T MR scanner (Siemens Healthcare, Erlangen, Germany).

We found that for the phantom brain tissue, PVA-C made with $6 \%$ PVA solution at 1 FTC containing concentration of $2 \% \mathrm{BaSO}_{4}, 0.025 \% \mathrm{CuSO}_{4}$, and $1 \%$ talcum as contrast agents worked well for $\mathrm{CT}, \mathrm{MR}$, and US, respectively. For our triple modality image markers, we found that a gel made from a $8 \%$ PVA solution with $5 \% \mathrm{BaSO}_{4}, 0.2 \% \mathrm{CuSO}_{4}$, and $5 \%$ talcum as contrast agents worked well for CT, MR, and US, respectively. Although, $\mathrm{CuSO}_{4}$ diffuses rather quickly out of the PVA-C, it did not affect the quality of the images greatly if they were acquired within the week when the phantom was built. The texture of the PVA-C did not change dramatically with the addition of these quantities of contrast agents.

\section{Image Acquisition}

Multimodality images with deformations were acquired for the phantom using the same models of MR, CT, and US imaging devices that we used for determining imaging contrast (See Fig. 1 $b, c$ ). The phantom was first scanned in the MR with $\mathrm{T} 1$ weighted spin-echo $\left(\mathrm{TR}=668 \mathrm{~ms}, \mathrm{TE}=8.9 \mathrm{~ms}\right.$, Flip Angle $\left.=70^{\circ}, 1 \times 1 \times 3 \mathrm{~mm}\right)$ and gradient-echo imaging $\left(\mathrm{TR}=1900 \mathrm{~ms}, \mathrm{TE}=3 \mathrm{~ms}\right.$, Flip Angle $=9^{\circ}, 1 \mathrm{~mm}$ isotropic $)$, $\mathrm{T} 2$ weighted imaging $\left(\mathrm{TR}=6530 \mathrm{~ms}, \mathrm{TE}=840 \mathrm{~ms}\right.$, Flip Angle $\left.=150^{\circ}, 1 \times 1 \times 3 \mathrm{~mm}\right)$, proton density $\left(\mathrm{PD}: \mathrm{TR}=6530 \mathrm{~ms}, \mathrm{TE}=9.4 \mathrm{~ms}\right.$, Flip Angle $\left.=150^{\circ}, 1 \times 1 \times 3 \mathrm{~mm}\right)$, fluid attenuated inversion recovery (FLAIR: TR $=5000 \mathrm{~ms}, \mathrm{TE}=273 \mathrm{~ms}, \mathrm{TI}=1800 \mathrm{~ms}$, Flip Angle $=120^{\circ}, 1 \mathrm{~mm}$ isotropic), and 30 direction diffusion weighted (DWI: $\mathrm{TR}=9300 \mathrm{~ms}, \mathrm{TE}=94 \mathrm{~ms}$, Flip Angle $\left.=90^{\circ}, 1 \times 1 \times 2 \mathrm{~mm}\right)$ MR sequences. Fractional anisotropy, apparent diffusion coefficient, and trace weighted images were computed from the diffusion weighted images. A CT scan (491 axial slices at $1.25 \mathrm{~mm}$ thickness) was then acquired for the phantom followed by the acquisition of a series of tracked B-mode US images (44 images of 4-6 sweeps at each $5.2 \mathrm{~cm}$ and $7.1 \mathrm{~cm}$ depth).

All of the images from each modality were acquired one after the other to ensure that the phantom is in the same state in the set of images. After each series of multimodality imaging, the phantom was deformed by inflating each of the two implanted urinary catheters in the phantom with $0 \mathrm{ml}, 5 \mathrm{ml}$ or $10 \mathrm{ml}$ of water through 5 rounds of inflations (See Fig. 2).

As well, images for super-resolution image processing were acquired by scanning the phantom with MP-RAGE T1 weighted gradient echo sequence 6 times at an isotropic resolution of $0.5 \mathrm{~mm}$ with a slight displacement of the phantom less than $1 \mathrm{~cm}$ between each acquisition. The phantom was then scanned using a $\mathrm{T} 1$ weighted spin echo sequence at $1 \mathrm{~mm}$ isotropic resolution 7 times with its container filled with water and 7 times without the water, again with a slight displacement of the phantom less than $1 \mathrm{~cm}$ between each acquisition. 


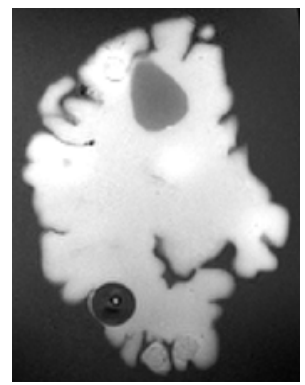

(a)

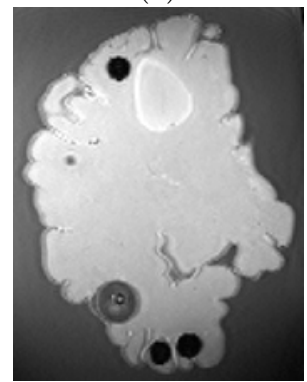

(e)

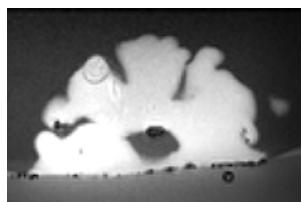

I

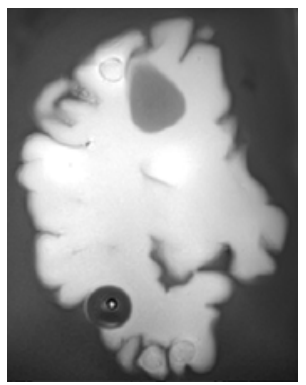

(b)

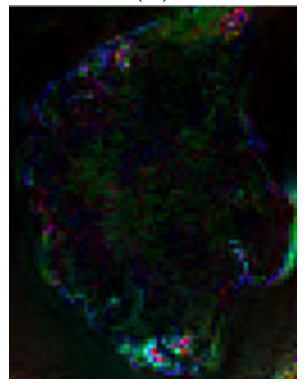

(f)

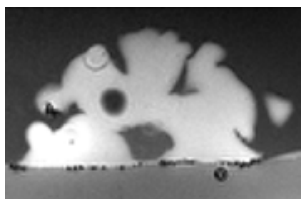

II

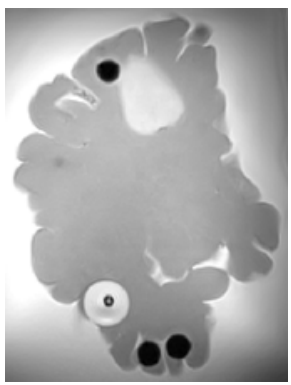

(c)

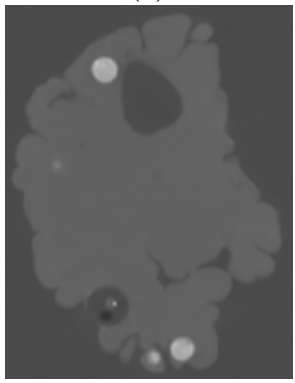

(g)

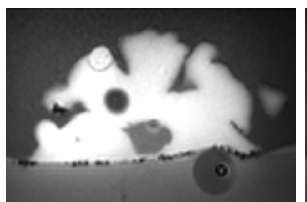

III

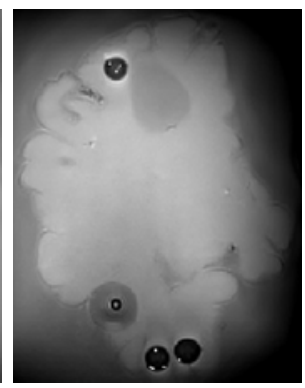

(d)

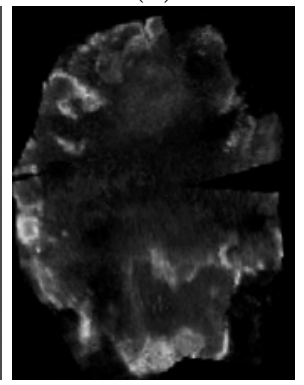

(h)

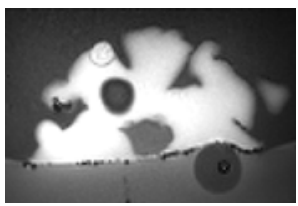

IV

Fig. 2. A selection of PVA-C brain phantom images acquired using (a) MR T1-weighted gradient-echo (b) MR T1-weighted spin-echo (c) MR T2-weighted (d) MR PD (e) MR FLAIR (f) MR DTI colour map (g) CT (h) and the reconstructed US sweeps. Images I-IV shows the phantom imaged with T1-weighted gradient-echo at different inflations of the catheters.

\section{Conclusion}

An anatomically and mechanically realistic PVA-C brain phantom was created and imaged using MR, CT, and US. The images acquired from this phantom were then made publically available to the larger image processing community. We believe that the acquired multi-modal images be used for validation of many image processing techniques such as segmentation, super-resolution, image reconstruction, linear or non-linear registration, and denoising algorithms, using images acquired from one modality to act as the ground truth of another. The images are made available at: http://pvabrain.inria.fr 
Aside from image processing, the formulation of our phantom material to approximate live cerebral brain tissue can be invaluable for improving implantation for deep-brain stimulators and simulating biopsy needle insertions. The accurate anatomy and texture of the brain phantom as well as the low cost of the starting materials can also make it useful as a tool in training medical professionals.

In order to further improve the phantom, we are currently in the process of developing better multimodal imaging spherical markers for higher contrast imaging, and finding better MR contrast agents that do not diffuse. We would also like to further characterize the multi-modal PVA-C developed in the work such that the physical and imaging property of the material can be better understood. Nevertheless, the presented methods represent an important step in the development of multimodality imageable tissue-like materials, along with techniques for creating anatomically accurate brain phantoms.

Acknowledgements. We would like to thank the Neurimage platform team for its financial support and their assistance in processing and acquiring the MR data, as well as Professor Olivier Le Guillou and Florence Le Dret at the chemistry department of the Institut National des Sciences Appliquées (INSA) de Rennes for allowing us to use their equipment for preparing solutions. We are also grateful to the Fonds québécois de la recherche sur la nature et les technologies (FQRNT) and the Collège Doctoral International (CDI) de l'Université européenne de Bretagne (UEB) Bourse de Mobilité Internationale Entrante for funding this project.

\section{References}

1. Holmes, C., Hoge, R., Collins, L., Woods, R., Toga, A., Evans, A.: Enhancement of MR images using registration for signal averaging. Journal of Computer Assisted Tomography 22(2), 324 (1998)

2. Surry, K.J.M., Austin, H.J.B., Fenster, A., Peters, T.M.: Poly(vinyl alcohol) cryogel phantoms for use in ultrasound and $\mathrm{mr}$ imaging. Physics in Medicine and Biology 49(24), 5529-5546 (2004)

3. Reinertsen, I., Descoteaux, M., Drouin, S., Siddiqi, K., Collins, D.L.: Vessel driven correction of brain shift. In: Barillot, C., Haynor, D.R., Hellier, P. (eds.) MICCAI 2004. LNCS, vol. 3217, pp. 208-216. Springer, Heidelberg (2004)

4. Reinertsen, I., Collins, D.L.: A realistic phantom for brain-shift simulations. Medical Physics 33(9), 3234-3240 (2006)

5. Peppas, N.A.: Turbidimetric studies of aqueous poly(vinyl alcohol) solutions. Die Makromolekulare Chemie 176(11), 3433-3440 (1975)

6. Stauffer, S.R., Peppas, N.A.: Poly (vinyl alcohol) hydrogels prepared by freezingthawing cyclic processing. Polymer 33(18), 3932-3936 (1992)

7. Peppas, N.A., Stauffer, S.R.: Reinforced uncrosslinked poly (vinyl alcohol) gels produced by cyclic freezing-thawing processes: a short review. Journal of Controlled Release 16(3), 305-310 (1991)

8. Chu, K.C., Rutt, B.K.: Polyvinyl alcohol cryogel: An ideal phantom material for MR studies of arterial flow and elasticity. Magnetic Resonance in Medicine 37(2), 314-319 (1997) 
9. Duboeuf, F., Bbasarab, A., Liebgott, H., Brusseau, E., Delechartre, P., Vray, D.: Investigation of pva cryogel young's modulus stability with time, controlled by a simple reliable technique. Medical Physics 36(2), 656-661 (2009)

10. Mehrabian, H., Samani, A.: Constrained hyperelastic parameters reconstruction of pva (polyvinyl alcohol) phantom undergoing large deformation. In: Medical Imaging 2009: Visualization, Image-Guided Procedures, and Modeling, vol. 7261 (March 2009)

11. Manohar, S., Kharine, A., van Hespen, J.C.G., Steenbergen, W., van Leeuwen, T.G.: Photoacoustic mammography laboratory prototype: imaging of breast tissue phantoms. Journal of Biomedical Optics 9(6), 1172-1181 (2004)

12. Fromageau, J., Gennisson, J.L., Schmitt, C., Maurice, R., Mongrain, R., Cloutier, G.: Estimation of polyvinyl alcohol cryogel mechanical properties with four ultrasound elastography methods and comparison with gold standard testings. IEEE Transactions on Ultrasonics, Ferroelectrics and Frequency Control 54(3), 498-509 (2007)

13. Khaled, W., Neumann, T., Ermert, H., Reichling, S., Arnold, A., Bruhns, O.: Evaluation of material parameters of pva phantoms for reconstructive ultrasound elastography. In: IEEE Ultrasonics Symposium, pp. 1329-1332 (2007)

14. Jia, C., Kim, K., Kolias, T., Weitzel, W., Rubin, J., O’Donnell, M.: Left ventricular phantom with pulsatile circulation for ultrasound strain rate imaging. In: IEEE Ultrasonics Symposium, pp. 1317-1320 (2006)

15. Fallenstein, G., Hulce, V., Melvin, J.: Dynamic mechanical properties of human brain tissue. Journal of Biomechanics 2(3), 217-226 (1969) 